\title{
Molecular mechanisms of Cys-loop ion channel receptor modulation by ivermectin
}

\author{
Timothy Lynagh ${ }^{1 \dagger}$ and Joseph W. Lynch ${ }^{1,2 *}$ \\ Queensland Brain Institute, The University of Queensland, Brisbane, QLD, Australia \\ 2 School of Biomedical Sciences, The University of Queensland, Brisbane, QLD, Australia
}

\section{Edited by:}

Piotr Bregestovski, Institut National de la Santé et de la Recherche

Médicale, France

\section{Reviewed by:}

Neil S. Millar, University College

London, UK

Stanko S. Stojilkovic, National

Institutes of Health, USA

\section{*Correspondence:}

Joseph W. Lynch, Queensland Brain

Institute, The University of

Queensland, Brisbane, OLD 4072,

Australia.

e-mail: j.lynch@uq.edu.au

${ }^{\dagger}$ Present address:

Department of Molecular and

Cellular Neurophysiology, Technical

University of Darmstadt, Germany.
Ivermectin is an anthelmintic drug that works by inhibiting neuronal activity and muscular contractility in arthropods and nematodes. It works by activating glutamate-gated chloride channels (GluCIRs) at nanomolar concentrations. These receptors, found exclusively in invertebrates, belong to the pentameric Cys-loop receptor family of ligand-gated ion channels (LGICs). Higher (micromolar) concentrations of ivermectin also activate or modulate vertebrate Cys-loop receptors, including the excitatory nicotinic and the inhibitory GABA type-A and glycine receptors (GlyRs). An X-ray crystal structure of ivermectin complexed with the $C$. elegans $\alpha$ GluCIR demonstrated that ivermectin binds to the transmembrane domain in a cleft at the interface of adjacent subunits. It also identified three hydrogen bonds thought to attach ivermectin to its site. Site-directed mutagenesis and voltage-clamp electrophysiology have also been employed to probe the binding site for ivermectin in $\alpha 1$ GlyRs. These have raised doubts as to whether the hydrogen bonds are essential for high ivermectin potency. Due to its lipophilic nature, it is likely that ivermectin accumulates in the membrane and binds reversibly (i.e., weakly) to its site. Several lines of evidence suggest that ivermectin opens the channel pore via a structural change distinct from that induced by the neurotransmitter agonist. Conformational changes occurring at locations distant from the pore can be probed using voltage-clamp fluorometry (VCF), a technique which involves quantitating agonist-induced fluorescence changes from environmentally sensitive fluorophores covalently attached to receptor domains of interest. This technique has demonstrated that ivermectin induces a global conformational change that propagates from the transmembrane domain to the neurotransmitter binding site, thus suggesting a mechanism by which ivermectin potentiates neurotransmitter-gated currents. Together, this information provides new insights into the mechanisms of action of this important drug.

Keywords: Cys-loop receptor, chloride channel, anthelmintic, avermectin, nematode, parasite

\section{INTRODUCTION}

Ligand-gated ion channels (LGICs) of the pentameric Cys-loop receptor family mediate most of the fast synaptic neurotransmission in the brain. The binding of a neurotransmitter ligand to the extracellular domain of the Cys-loop receptor triggers the opening of an intrinsic ion channel in its membrane-spanning domain, allowing ions to diffuse down their electrochemical gradients across the membrane (Changeux, 2010). As nicotinic acetylcholine receptors (nAChRs) and type-3 5-hydroxytryptamine receptors $\left(5-\mathrm{HT}_{3} \mathrm{Rs}\right)$ are selective for cations, their activation leads to depolarization and neuronal excitation. Conversely, as the pores of receptors for $\gamma$-aminobutyric acid and glycine $\left(\mathrm{GABA}_{\mathrm{A}} \mathrm{Rs}\right.$ and GlyRs) are selective for chloride, their activation will tend to move the membrane potential rapidly toward the chloride equilibrium potential. Because this lies near to the resting potential in adult neurons, chloride channel activation tends to limit the ability of excitatory stimuli to depolarise the neuron. Drugs that enhance the flux of chloride through inhibitory Cysloop receptors therefore inhibit neuronal activity in the brain or spinal cord (Rudolph and Antkowiak, 2004).
Invertebrates often express a larger and more diverse array of inhibitory Cys-loop receptor subunits than vertebrates (Jones and Sattelle, 2008; Jones et al., 2010). For example, numerous protostomes express glutamate-gated chloride channel receptors (GluClRs), which are absent in vertebrates (Kehoe et al., 2009). Hence, avermectins and milbemycins, which potently activate GluClRs, lethally depress the nervous and muscular system of GluClR-possessing species (Wolstenholme and Rogers, 2005), and the gold standard avermectin anthelmintic, ivermectin, is a well-tolerated and remarkably successful drug for eliminating parasitic nematodes and insects from mammals (Omura, 2008). Ivermectin also activates vertebrate GlyRs and $\mathrm{GABA}_{\mathrm{A}} \mathrm{Rs}$, although less potently than GluClRs (Adelsberger et al., 2000; Shan et al., 2001). Interestingly, its activation of Cys-loop receptors is mechanistically distinct to that of neurotransmitter agonists (Hibbs and Gouaux, 2011). Whereas the latter activate in microseconds currents that are rapidly reversible, early tests with ivermectin showed currents developed over a period of seconds and were irreversible (Fritz et al., 1979; Kass et al., 1980). The distinct actions of ivermectin make it useful for several reasons. 
It can be used to target vertebrate Cys-loop receptors for novel therapeutic leads for a variety of indications. For example, drugs that enhance the activity of GlyRs may be useful for treating disorders such as spasticity, epilepsy, tinnitus, breathing disorders, and chronic pain (Costa and Diazgranados, 1994; Webb and Lynch, 2007; Eichler et al., 2009; Yevenes and Zeilhofer, 2011). Secondly, receptors engineered to eliminate neurotransmitter sensitivity and increase ivermectin sensitivity may be useful for the pharmacogenetic control of neurons in vivo (Lerchner et al., 2007; Lynagh and Lynch, 2010b). Finally, an understanding of ivermectin binding and activation mechanisms may be useful for understanding the molecular basis of ivermectin resistance in nematode parasite pests (Wolstenholme et al., 2004; Wolstenholme, 2010; Ghosh et al., 2012). Our understanding of ivermectin binding and activation mechanisms has advanced rapidly in the past 12 months, with the publication of a crystal structure of ivermectin bound to the C. elegans $\alpha$ GluClR (Hibbs and Gouaux, 2011) and a systematic site-directed mutagenesis study on ivermectin sensitivity determinants at the $\alpha 1$ GlyR (Lynagh et al., 2011). Thus, it is timely to bring together the knowledge of how ivermectin modulates these channels, in order to facilitate the development of ivermectin-based therapies and neuronal silencing tools.

Consistent with this review forming part of a series entitled "Molecular probes and switches for functional analysis of receptors, ion channels and synaptic networks," we have focused on the use of fluorescent techniques to probe conformational changes elicited by ivermectin in Cys-loop ion channel receptors.

\section{Cys-LOOP RECEPTOR STRUCTURE AND FUNCTION}

The Cys-loop receptor family is diverse, with excitatory receptors for acetylcholine, 5-hydroxytryptamine (5-HT), GABA and zinc, and inhibitory receptors for acetylcholine, 5-HT, GABA, glycine, glutamate, histamine, and even pH (Cully et al., 1994; Ranganathan et al., 2000; Zheng et al., 2002; Beg and Jorgensen, 2003; Schnizler et al., 2005; Collingridge et al., 2009). However, the tertiary structures and overarching mechanisms of activation appear to be highly conserved. Before detailing the molecular mechanisms of ivermectin action on these receptors, it will be useful to understand the common structural and functional features of the family.

Functional Cys-loop receptors are formed by the assembly of five of the same (homomer) or different (heteromer) subunits (Figures 1A,B). Each subunit consists of a large extracellular N-terminal domain (ECD), four membrane-spanning helices (M1-M4) that constitute the transmembrane domain (TMD) and a short extracellular C-terminal domain. The ECD consists of $10 \beta$-strands; the loop between the sixth and seventh is constrained by a disulfide bond between two highly conserved cysteine residues, giving the family its name. The subunits are arranged with five-fold symmetry, such that the M2 helices from each subunit are located centrally, forming the channel pore (Figure 1). The amino acid composition of the M2 helix thus determines the ion selectivity and conductance properties of the channel (Imoto et al., 1988; Galzi et al., 1992; Keramidas et al., 2000, 2002). To facilitate the comparison of different subunits, a common numbering system is used for M2 amino acid positions, starting with $0^{\prime}$, a highly conserved positively charged amino acid near the intracellular C-terminal end. Moving outwards through the pore, positions $2^{\prime}, 6^{\prime}, 9^{\prime}, 13^{\prime}$, and $16^{\prime}$ line the pore of most Cys-loop receptor channels, according to their accessibility to hydrophilic reagents and recent crystal structures (Akabas et al., 1994; Cymes et al., 2005; Hibbs and Gouaux, 2011). At the $-1^{\prime}$ position, cationic Cys-loop channels generally possess a negatively charged amino acid, whereas anionic channels possess a neutral residue (Galzi et al., 1992).

\section{NEUROTRANSMITTER ACTIVATION MECHANISMS}

By the early 1990's the genes for most human Cys-loop receptor subunits had been cloned, enabling the expression of recombinant receptors of known identity in mammalian cells and oocytes. This in turn facilitated their examination by patch-clamp electrophysiology (in the case of mammalian cells) or two-electrode voltage-clamp electrophysiology (in oocytes). By mutating particular amino acids and testing for loss or gain of function, much knowledge has accumulated on the molecular mechanisms of ligand binding, ionic selectivity, and receptor gating. Interpretation of these functional data is now aided by X-ray crystallographic structures of molluscan acetylcholine binding proteins (AChBPs), pentamers that resemble the ECD of the nAChR (Brejc et al., 2001), a GluClR crystal structure from C. elegans (Hibbs and Gouaux, 2011) and an electron micrographic structure of the nAChR (Unwin, 2005). X-ray crystallographic data on two presumably closed- and open-state bacterial homologs, ELIC, and GLIC, also inform hypotheses on Cys-loop receptor activation mechanisms (Hilf and Dutzler, 2008; Bocquet et al., 2009). In the metazoan receptors, the agonist binding site is at the interface of ECDs from two adjacent subunits. It is formed by three loops A-C of the principal face and three to four loops (or rather $\beta$-strands) $\mathrm{D}-\mathrm{G}$ of the complementary face (Figure 1C). Substitutions of residues in these loops in nAChRs, 5-HT $\mathrm{H}_{3}$, $\mathrm{GABA}_{\mathrm{A}}$ Rs, GlyRs, and GluClRs thus cause large decreases in agonist sensitivity (Galzi et al., 1991; Amin and Weiss, 1993; Rajendra et al., 1995; Li et al., 2002; Hazai et al., 2009). Several important ligand-binding residues are indicated in green in Figure 1. Following ligand binding, a wave of conformational changes proceeds away from the binding site toward the channel gate (Purohit et al., 2007) and numerous mutagenesis studies have implicated amino acids involved in mediating these conformational changes (Miller and Smart, 2010; Thompson et al., 2010).

Cysteine cross-linking in the $\mathrm{GABA}_{\mathrm{A}} \mathrm{R}$ ECD and analysis of $\mathrm{AChBP}$ and $\mathrm{nAChR}$ structures in liganded and unliganded conformations suggest that upon ligand binding, loops $\mathrm{C}$ and $\mathrm{B}$ of the principal ligand binding face move toward the complementary face and around the agonist (Wagner and Czajkowski, 2001; Hansen et al., 2005; Unwin, 2005). Structural analyses and molecular dynamics simulations suggest that this clockwise movement of ECDs (when the pentamer is viewed from above, as in Figure 1A) is coupled to a counter-clockwise rotation of TMDs (Taly et al., 2005; Bocquet et al., 2009). There are several obvious physical pathways along which the ECD movement might cause TMD movement. $\beta$-strand 10, which forms Loop C 

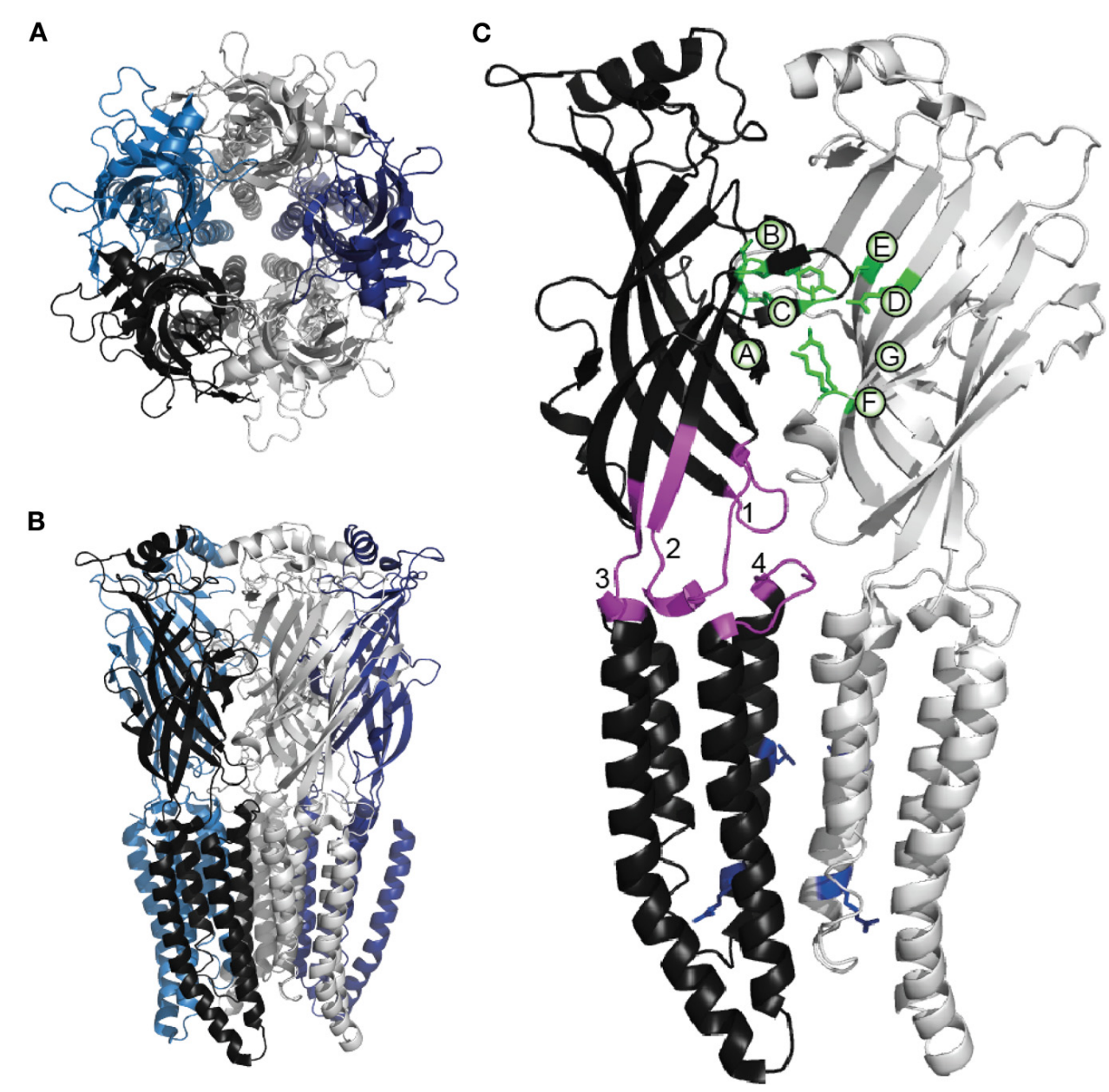

FIGURE 1 | Cys-loop receptor structure. The homomeric $\alpha$ GluCIR from C. elegans viewed (A) from the extracellular space and (B) from within the membrane plane. Individual subunits are shown in different colors.

(A) depicts the symmetrical arrangement of both the extracellular domains and, centrally, of the M2 helices. These images are based on the original X-ray crystallographic structure of $3.3 \AA$ resolution (PDB ID 3RHW), which was achieved by complexing the GluCIR with an antigen-binding fragment, ivermectin and lipids, and represents an activated, open-channel state (Hibbs and Gouaux, 2011). The present images were produced using PyMOL

(Schrödinger, LLC). (C) Two adjacent subunits from (B), indicating functionally

significant parts of the Cys-loop receptor subunit. Green side chains indicate a $\mathrm{GluCl}$ residues that bind the agonist glutamate (Hibbs and Gouaux, 2011). As in most Cys-loop receptors, the agonist binding residues are located in loops A-C from the principal subunit and loops D-G from the complementary subunit, as indicated. Within the principal subunit, loop $2(1)$, the conserved Cys-loop (2), the pre-M1 domain (3), and the M2-M3 linker (4) are shown in magenta; these structures physically couple agonist binding to channel opening (see text). Blue side chains in both subunits indicate conserved $9^{\prime}$ hydrophobic residues (upper) and the $0^{\prime}$ basic residues (lower) that control channel conductance and ionic selectivity.

with $\beta$-strand 9, is linked via the "pre-M1 domain" to M1 of the TMD (Figure 1). Loops D and G ( $\beta$-strands 2 and 1 ) form "loop 2 " which dips toward the TMD, making close contact with the "M2-M3 linker." Finally, the conserved Cys-loop that connects Loops E and B ( $\beta$-strands 6 and 7 ) dips well into the helical bundle of the individual subunit. Mutations in these domains often alter the ability of bound ligand to activate the channel, because they disrupt a specific set of ionic and hydrophobic links formed across the interface of the ECD and TMD (Kash et al., 2003; Grutter et al., 2005; Lee and Sine, 2005; Xiu et al., 2005; Pless et al., 2011).

The closed channel is narrowest near its middle, where the side chains from highly conserved M2 9' leucine residues form a hydrophobic girdle (Miyazawa et al., 2003; Hilf and
Dutzler, 2008). In defining how this gate is opened, various groups have suggested that the M2 helices either rotate (Unwin, 1995; Horenstein et al., 2001), move apart without rotating (Akabas et al., 1994; Cymes et al., 2005; Paas et al., 2005) or tilt tangentially to the pore (Taly et al., 2005). Static pictures of the closed and open states, as revealed by crystal structures, show the upper halves of the M2 helices transitioning away from the pore axis in the open state, such that the pore restriction remains at the $-2^{\prime}$ and $+2^{\prime}$ side chains (Bocquet et al., 2009; Hilf and Dutzler, 2009; Hibbs and Gouaux, 2011). This seems consistent with the role of this region in forming the ion selectivity filter because the charge filtering property of the pore is critically dependent upon its diameter (Galzi et al., 1992; Keramidas et al., 2004; Cymes and Grosman, 2011). It is important to 
note, however, that these crystal structures depict ivermectin- or proton-bound receptors, neither of which is a conventional ligand. Thus, an agreement on the structures of the resting closed and the transmitter-induced open states, or the mechanism by which the channel transitions from one state to the other, has not been reached.

\section{THE IVERMECTIN BINDING SITE IN Cys-LOOP RECEPTORS}

Ivermectin comprises a mixture of 22,23-dihydroavermectin B1a and $\mathrm{B} 1 \mathrm{~b}$ in a 80:20 ratio. These compounds differ in structure only at C25, a part of the molecule not involved in binding. Given the greater abundance and nematicidal efficacy of avermectin B1a (Crump and Omura, 2011), it is conventional to refer to the above mixture as "ivermectin" and this usage will be adopted here. Ivermectin directly activates GlyRs, GluClRs, and GABA Rs, generally via a mechanism that is either irreversible or very slowly reversible (Cully et al., 1994; Etter et al., 1996; Adelsberger et al., 2000; Shan et al., 2001; Hibbs and Gouaux, 2011). In addition, at concentrations that are too low to produce direct activation, ivermectin also potently enhances neurotransmitter-gated currents (Cully et al., 1994; Huang and Casida, 1997; Shan et al., 2001). Ivermectin has not been shown to influence $5-\mathrm{HT}_{3} \mathrm{R}$ activity, although at high $(30 \mu \mathrm{M})$ concentrations it potentiates $\alpha 7$ nAChRs (Krause et al., 1998; Raymond et al., 2000; Collins and Millar, 2010).

It was first proposed many years ago that ivermectin might interact with the TMD channel domain of Cys-loop receptors (Pong and Wang, 1982; Martin and Kusel, 1992). However, the molecular determinants of ivermectin binding have only recently been identified. Hibbs and Gouaux have described at high resolution ivermectin bound to the homomeric $\alpha \mathrm{GluClR}$ from C. elegans (Hibbs and Gouaux, 2011). Ivermectin binds at the TMD interface of two adjacent subunits, wedged between M3 from the principal subunit ("M3(+)") and M1 from the complementary subunit ("M1(-)") (Figure 2A). The benzofuran head of ivermectin is orientated toward the pore, such that the C5-hydroxyl of ivermectin forms a hydrogen bond with the hydroxyl side chain of a serine residue at the $\mathrm{M} 2(+) 15^{\prime}$ position (Figure 2B). The C7-hydroxyl of ivermectin forms a hydrogen bond with the backbone carbonyl of an M1 leucine (218) residue, but is also close enough to the carbonyl oxygen at the $\mathrm{Cl}$ position of ivermectin to form a hydrogen bond with the latter (Figure 2B). The spiroketal is oriented toward the cytoplasm with a hydrogen bond between a spiroketal oxygen and the hydroxyl side chain of an M3 threonine (285). The disaccharide remains outside the binding cleft in contact with the surrounding lipids and the protein surface. There are an additional twelve van der Waals interactions between ivermectin and M3 $(+), M 2(+)$, and $\mathrm{M} 1(-)$ residues.

Mutagenesis experiments, combined with an homology model based on the $C$. elegans $\alpha$ GluClR, reveal a similar binding orientation for ivermectin in GlyRs. In the $\alpha 1$ GlyR, M3(+) Ala288Phe and $\mathrm{M1}(-)$ Pro230Trp mutations were unique in that they decreased both the direct agonist and potentiating effects of ivermectin, singling out these two residues as possible determinants of ivermectin binding (Lynagh et al., 2011). Indeed, Ala288 and Pro230 are equivalent to two of the $\alpha$ GluClR residues identified

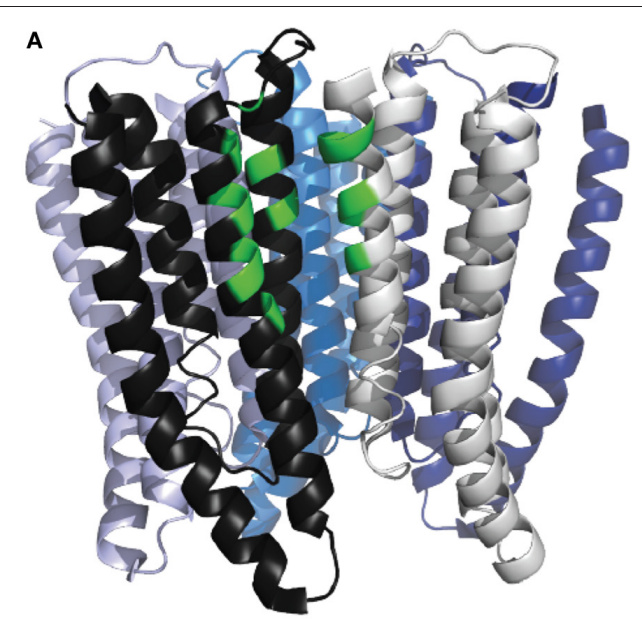

B

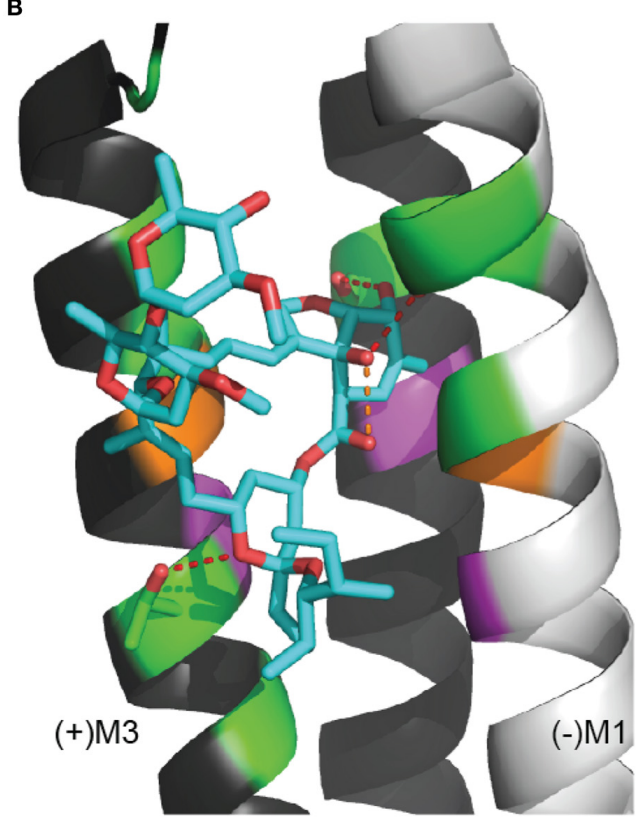

$(+) \mathrm{M} 2$

FIGURE 2 | The ivermectin binding site in the $C$. elegans $\alpha$ GluCIR crystal structure. (A) The GluCIR pentamer viewed from within the membrane plane, with ECDs removed for clarity. At one subunit interface, the residues that contact the bound ivermectin molecule are indicated in green, showing that ivermectin binds to the receptor in the outer half of the membrane. (B) Ivermectin (blue) bound to a subunit interface. Ivermectin forms hydrogen bonds (red dashed lines) with (+)M3 threonine 285, $(+) \mathrm{M} 2$ serine $15^{\prime}$ and the backbone carbonyl oxygen of (-)M1 leucine 218 (Hibbs and Gouaux, 2011). The orange dashed line indicates the $2.6 \AA$ proximity of the $\mathrm{C} 7$ hydroxyl oxygen and the $\mathrm{C} 1$ carbonyl oxygen of ivermectin. Green indicates residues proximal to ivermectin, indicative of hydrogen bonds or van der Waals interactions. Purple indicates residues equivalent to those in the $\alpha 1$ GlyR (GlyR M3 leucine 291, M2 threonine 12', M1 leucine 233) whose substitution by tryptophan causes ivermectin to act as an inhbitor (Lynagh et al., 2011). Orange indicates residues (GluCIR (+)M3 glycine 285 and (-)M1 223) that contribute van der Waals interactions in the GluCIR and whose substitution for larger residues decreases ivermectin sensitivity in the $\alpha 1$ GlyR (the (-)M1 residue) or in both $\alpha 1$ GlyR and the $H$. contortus $\alpha 3 \mathrm{~B} \mathrm{GluCl}$ (the (+)M3 residue) (Lynagh and Lynch, 2010b; Lynagh et al., 2011). This figure uses the same PDB file and software as Figure 1 
by Hibbs and Gouaux as being involved in van der Waals interactions with the ivermectin backbone macrocycle. Functional experiments have shown that mutations to Ala288 in the $\alpha 1$ GlyR, or to the corresponding residue in the $\alpha 3 \mathrm{~B}$ GluClR, have a particularly strong influence on ivermectin potency (Lynagh and Lynch, 2010a). For example, if a glycine residue exists at this site, as in the unmutated $\alpha 3 \mathrm{~B}$ GluClR or the Ala288Gly mutant $\alpha 1$ GlyR, the recombinant receptors show an ivermectin agonist $\mathrm{EC}_{50}$ around $40 \mathrm{nM}$. Alternately, if there is an alanine residue at this site, as in the Gly329Ala mutant $\alpha 3 \mathrm{~B}$ GluClR or in the unmutated $\alpha 1$ GlyR, the recombinant receptors show an $\mathrm{EC}_{50}$ of $1.2 \mu \mathrm{M}$. Larger substitutions at this position abolish activation in both receptors (Lynagh and Lynch, 2010a). The Gly323Asp mutation, at the equivalent position to Ala288, in the $\alpha$ GluClR of the acarid, Tetranychus urticae, results in avermectin resistance in this otherwise avermectin-sensitive species (Kwon et al., 2010). Surprisingly, computational docking of ivermectin to a structural homology model of the mutant Ala288Gly $\alpha 1$ GlyR predicts an ivermectin binding conformation almost identical to that at the unmutated $\alpha 1$ GlyR, suggesting that the ivermectin binding on-rate, rather than its binding energy, is altered by this mutation (Lynagh et al., 2011). Taken together with the site-directed mutagenesis information described above, this suggests large side chains at the Ala288 equivalent position constitute a barrier to ivermectin accessing its site, but once ivermectin does bind the conformation is not affected by the side chain at this position. The binding of tritiated milbemycin A4 (similar to ivermectin but without the disaccharide) to the $\alpha 3 \mathrm{~B}$ GluClR was recently shown to be abolished by the Gly-Asp at the position corresponding to Ala288 (Yamaguchi et al., 2012).

Hibbs and Gouaux suggested that ivermectin binding wedges apart $\mathrm{M} 3(+)$ and $\mathrm{M} 1(-)$ helices in such a way as to move the M2 helix from its resting closed state; the C5-hydroxyl/M2(+) $15^{\prime}$ hydrogen bond then serves to stabilise the M2 helix in its open state. This is a mechanistic explanation of refreshing tangibility. It could also explain the reduced nematicidal potency of avermectins lacking the C5-hydroxyl (Michael et al., 2001) and the finding that selamectin, which incorporates a C5-oxime, does not activate the GlyR (Lynagh et al., 2011). However, this and the other hydrogen bonds in the C. elegans $\alpha$ GluClR might not be conserved in other highly ivermectin-sensitive Cys-loop receptors. For example, potent ivermectin activation is still observed at the Ser 15' Ile mutant $\alpha 1$ GlyR (Lynagh et al., 2011) and at GluClRs that naturally incorporate non-hydrogen bonding side chains at the M2(+) 15' and/or M3(+) Thr285 ( $\alpha$ GluClR numbering) positions (Cully et al., 1996; Forrester et al., 2003; Njue et al., 2004; McCavera et al., 2009). Finally, C5-hydroxyl-substituted milbemycins do not suffer the same loss of potency as C5hydroxyl-substituted avermectins (Tsukamoto et al., 1991). It may be space at the $\mathrm{M} 3(+) / \mathrm{M} 1(-)$ interface, rather than hydrogen bonds, that most crucially determine avermectin and milbemycin potency.

Ivermectin also potentiates the P2X4 subtype of ionotropic ATP-gated ion channels, although it does not produce direct activation in the absence of ATP (Khakh et al., 1999). These receptors belong to a distinct structural family of ion channels that is comprised of three subunits. Tryptophan scanning mutagenesis of P2X4 receptors suggests that ivermectin binds at the lipidprotein interface, possibly in a cleft between adjacent transmembrane $\alpha$-helices (Silberberg et al., 2007). However, the location of the ivermectin binding site has not yet been addressed in the context of the recent crystal structure of a zebrafish $\mathrm{P} 2 \mathrm{X} 4$ receptor (Kawate et al., 2009).

\section{DOES IVERMECTIN BIND REVERSIBLY?}

There are two possible mechanisms to explain the apparent irreversible activation by ivermectin of GlyRs, GluClRs, and $\mathrm{GABA}_{\mathrm{A}}$ Rs. One is that it binds irreversibly, or covalently, to the receptor. In this scenario, low ivermectin concentrations should eventually occupy all sites and thus should eventually elicit maximal receptor activation. However, covalent interactions between ivermectin and its site are not evident in the GluClR crystal structure, nor would they be expected to exist given the chemical properties of ivermectin. The alternative explanation is that, due to its lipophilic nature, ivermectin partitions into the membrane where it reaches a high local concentration. Thus, much of the binding energy of ivermectin could derive from the non-specific free energy of membrane partitioning, with the actual ligandchannel interaction being quite weak and thus reversible (Lee and MacKinnon, 2004). Consistent with this model, ivermectin has been shown to partition into phospholipid vesicles with an estimated mole fraction partition coefficient of $10^{4}$ (Bloom and Matheson, 1993; Silberberg et al., 2007).

Single channel kinetic analysis is a direct method for assessing the reversibility of a binding interaction. Each time a neurotransmitter agonist binds to a Cys-loop receptor it induces a stochastic "burst" of single channel activity. The burst terminates once the agonist dissociates from the channel. Thus, analysis of both the burst duration and the inter-burst duration (i.e., the length of time between bursts) will allow one to determine the rates at which the agonist binds and unbinds from its site (Mortensen and Smart, 2007). It follows that if ivermectin binds irreversibly, then it should induce an irreversible burst of channel activity. Alternately, if ivermectin binds reversibly, then it should activate short stochastic bursts of activity. It may not be straightforward to discern between these mechanisms if multiple channels are present in a single patch. This is exemplified in a study of ivermectin on muscle membrane from the nematode Ascaris suum. Ivermectin initially induced the activation of a single channel, although the number of active channels increased over time to the extent that single channel activations could no longer be detected (Martin and Pennington, 1989). Adelsberger (Adelsberger et al., 2000) compared the effects of ivermectin and GABA at mammalian $\alpha 1 \beta 2 \gamma 2$ GABA $_{A}$ Rs. They showed by whole-cell recording that the rise-time and decay of ivermectin-activated currents were markedly slower than those of GABA-activated currents. Their single channel kinetic analysis revealed that ivermectin did not differ from GABA in terms of the two observed opening rate constants, although groups of openings were markedly longer in the presence of ivermectin. Thus, although ivermectin induces no desensitization and dissociates at a slower rate than GABA, ivermectin-induced single channel bursts were reversible, supporting the idea of ivermectin 
accumulating in the membrane and binding reversibly to the channel.

\section{IVERMECTIN ACTIVATION MECHANISMS}

Several lines of evidence suggest that ivermectin and glycine activate the GlyR via structurally distinct mechanisms. First, ivermectin potently activates GlyRs that are completely desensitized to glycine (Shan et al., 2001). Second, ivermectin- and glycine-mediated currents exhibit distinct pharmacological properties, implying different activated conformations (Shan et al., 2001; Pless et al., 2007). Third, mutations that eliminate glycine sensitivity frequently have little or no effect on ivermectin sensitivity and vice versa (Shan et al., 2001; Lynagh and Lynch, 2010a; Lynagh et al., 2011). Finally, voltage-clamp fluorometry (VCF) experiments, to be described in further detail below, show that conformational changes induced by the two ligands are distinct (Pless et al., 2007; Wang and Lynch, 2012). However, to date there has been no systematic attempt to integrate these observations into a structural model of ivermectin activation.

A mechanistic explanation as to why M3 movement should have an effect on M2 to open the channel has recently been suggested on the basis of experiments conducted on the bacterial Cys-loop receptor homolog, GLIC. Mutagenesis and molecular dynamics simulations of GLIC show that proton binding to an M2 histidine side chain strengthens interactions between M2 and M3 helices within a subunit (Wang et al., 2012). The authors propose that this enables the concerted rigid body movement of M2 and M3 helices which favors an open channel state, whereas in the proton-free closed channel state, M2 is decoupled from M3. Similarly, ivermectin binding to Cys-loop receptors might enable a similar coupling of M2 and M3 helices; if the wedging apart of M3(+) and M1(-) serves to "drag" M2 helices away from the pore axis in a concerted M3/M2 conformational change, this might provide an explanation for why mutations in the M2-M3 linker decrease the direct agonist potency and not the efficacy with which ivermectin potentiates neurotransmitter-gated currents (Kane et al., 2000; Lynagh et al., 2011). In the potentiation

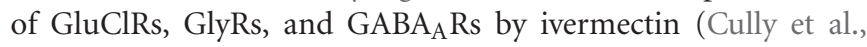
1994; Huang and Casida, 1997; Shan et al., 2001), wedging apart $\mathrm{M} 3(+)$ and $\mathrm{M} 1(-)$ might decrease the energy required for neurotransmitter agonists to effect M2 movement; in the activation of these channels, a subsequent coupling of ivermectin and M2 or of M2 and M3 might be required for direct activation. Similar mechanisms might underlie the potentiation and activation of $\mathrm{GABA}_{\mathrm{A}}$ Rs and GlyRs by the anaesthetic etomidate (Pistis et al., 1997), which binds in the same cleft as occupied by ivermectin (Li et al., 2006; Chiara et al., 2012).

If highly potent activation of the channel by ivermectin requires it to form a hydrogen bond with an $\mathrm{M} 2$ residue (Hibbs and Gouaux, 2011), then in most anionic Cys-loop receptors there are several alternate polar side chains other than $\mathrm{M} 2(+) 15^{\prime}$ that could be located close to the C5-hydroxyl of ivermectin to form such a bond. These include the M2(+) $12^{\prime}, \mathrm{M} 2(+) 19^{\prime}$, and the M2(-) $14^{\prime}$ positions. The locations of these residues in the ivermectin binding site are shown in Figure 3A and a sequence alignment showing the identity of these residues in various

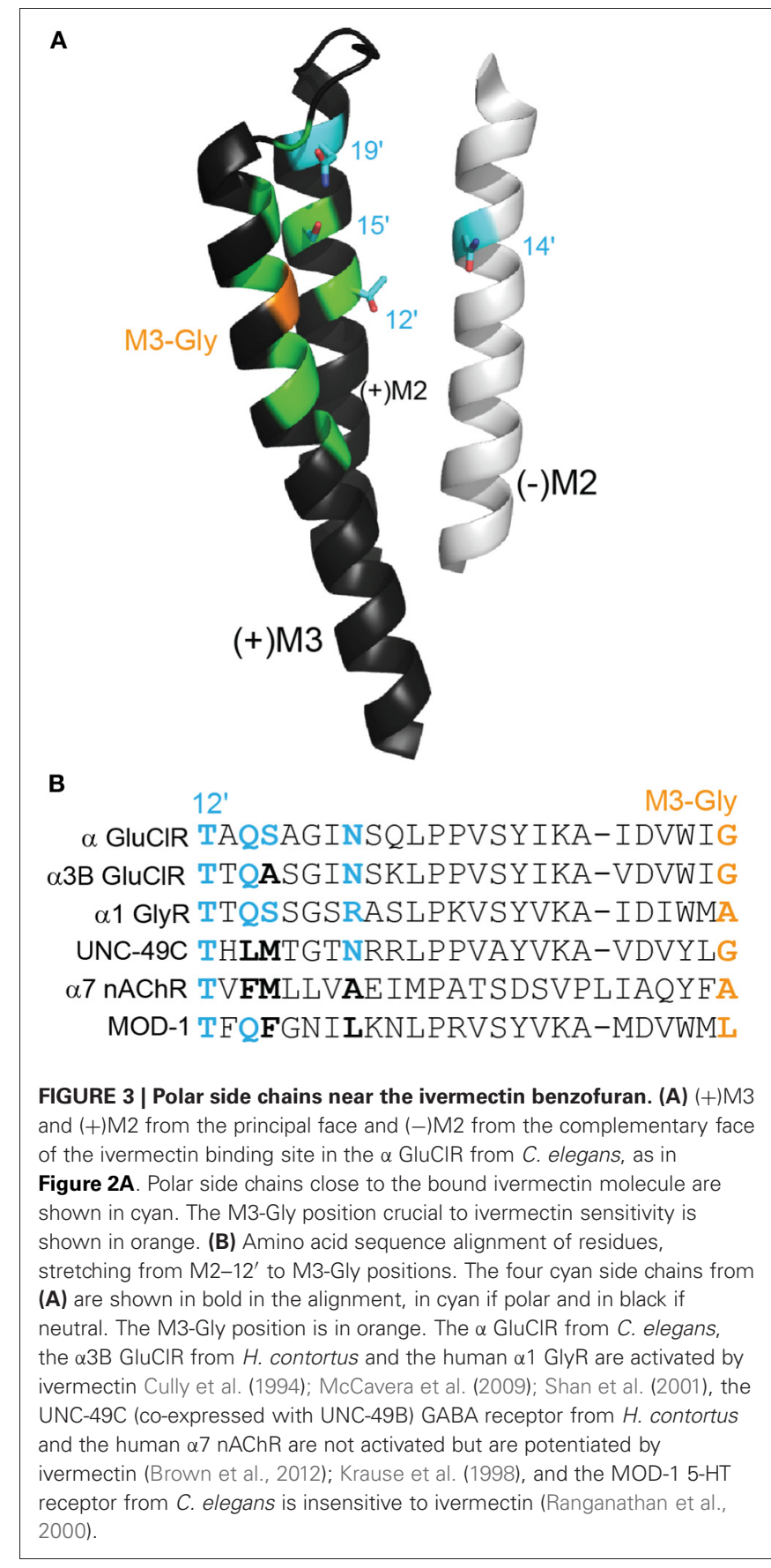

Cys-loop receptor subunits is shown in Figure 3B. Both displayed $\alpha$ GluClR subunits are sensitive to nanomolar ivermectin concentrations whereas the remaining subunits are not. As discussed above, the M3 glycine (shown in orange) is essential for high ivermectin sensitivity. It is also evident that polar sidechains at the positions indicated in blue (i.e., M2(+) 12', M2(-) $14^{\prime}, \mathrm{M} 2(+) 15^{\prime}$, and M2(+) $\left.19^{\prime}\right)$ are essential for high ivermectin sensitivity. Hydrophobic residues (in black) are incompatible with high ivermectin sensitivity, even if an M3-Gly is present. Substitutions at any of these positions in the $\alpha 1$ GlyR either convert ivermectin to an inhibitor of glycine currents or alter 
ivermectin agonist potency (Shan et al., 2001; Lynagh et al., 2011). It is also notable that $\alpha 7 \mathrm{nAChRs,} \mathrm{which} \mathrm{are} \mathrm{potentiated} \mathrm{but} \mathrm{not}$ activated by ivermectin (Krause et al., 1998; Raymond et al., 2000; Collins and Millar, 2010), possess neutral side chains at the 14', $15^{\prime}$, and $19^{\prime}$ positions (Figure 3B).

Maximal activation of Cys-loop channels may require as few as two bound ivermectin molecules. Perhaps the strongest evidence for this comes from experiments on $\alpha 1 \beta$ heteromeric GlyRs which most likely exist in a $\beta-\alpha 1-\beta-\alpha 1-\beta$ subunit arrangement (Grudzinska et al., 2005). When ivermectin binding to the $\alpha 1$ subunit was ablated by the Ala288Phe mutation, heteromeric receptors were completely insensitive to ivermectin (Lynagh and Lynch, 2010a). However, unmutated $\alpha 1 \beta$ heteromers exhibit a similar ivermectin potency to $\alpha 1$ homomeric GlyRs (Shan et al., 2001; Lynagh and Lynch, 2010a). This implies that ivermectin maximally activates heteromeric GlyRs by binding only to the two $\alpha(+) / \beta(-)$ interfaces (Lynagh and Lynch, 2010a).

\section{VOLTAGE-CLAMP FLUOROMETRY TO DETECT IVERMECTIN-INDUCED CONFORMATIONAL CHANGES IN THE ECD}

As described above, ivermectin must rearrange the TMD helical organization in order to open the channel. This rearrangement is in turn likely to induce a conformational change in the M2-M3 loop. If this is the case then mutation to the M2-M3 loop, or to the ECD domains that interact closely with it, might be expected to affect ivermectin gating efficacy. Indeed, mutations in the conserved Cys-loop, the M2-M3 linker and the pre-M1 region of GlyRs and GluClRs have been shown to decrease the efficacy with which ivermectin gates the receptor (Kane et al., 2000; Lynagh et al., 2011). At the GlyR, these mutations typically increased ivermectin agonist $\mathrm{EC}_{50}$ values 7 - to 14 -fold without affecting the potency with which ivermectin potentiated glycine-mediated currents (Lynagh et al., 2011). This suggests that the mutations did not disrupt ivermectin affinity, but rather the efficacy with which it activated the receptor. Indeed, such mutations have been shown to alter ivermectin and milbemycin binding per se to the a3B GluClR (McCavera et al., 2009; Yamaguchi et al., 2012). Given that ivermectin induces conformational changes at the ECD-TMD interface, it is possible that it may "globally" alter the conformation of the entire ECD. Understanding the structural basis of these conformational changes may have implications for understanding the mechanism by which ivermectin potentiates neurotransmitter-gated currents (Cully et al., 1994; Huang and Casida, 1997; Shan et al., 2001) and permits glutamate to bind to the $\alpha$ GluClR (Hibbs and Gouaux, 2011). Voltage- VCF is one useful means of comparing glycine- and ivermectin-induced conformational changes in the ECD.

VCF involves covalently attaching an environmentally sensitive fluorophore to a receptor domain of interest. The first step is to engineer a cysteine into the site of interest in an otherwise cysteine-free protein. The protein, which is usually recombinantly expressed in Xenopus oocytes, is then reacted with a fluorescent reporter conjugated to a sulfhydryl-reactive group, normally a methanethiosulfonate (MTS) or maleimide. Rhodamine derivatives appear to be retaining their popularity as the most widely used fluorophores for these types of experiments. However, many other fluorophores have been successfully used, and fluorophore selection is largely a matter of trial and error (Pless and Lynch, 2008). In our laboratory, we routinely employ four fluorophores and generally only analyse results from one that gives the large fluorescence response at a given labeled site (Pless and Lynch, 2009; Wang and Lynch, 2012).

Although the choice of light source may depend on the spectral properties of the fluorophore, we find that a 100-150 watt halogen lamp gives best results. Xenon and mercury burners can also be used, although these generate increased light noise and their intensity is not as easily controlled. A standard inverted epifluorescence microscope is suitable for most VCF applications (Pless and Lynch, 2008). Electrophysiology can be performed using standard two-electrode voltage-clamp and fluorescence signals can be measured simultaneously using a photomultiplier or photodiode. VCF takes advantage of the fact that the quantum yield of many organic fluorophores is highly sensitive to the chemical nature of their environment. For example, the fluorescence intensity of rhodamine increases with hydrophobicity (Gandhi and Isacoff, 2005). Thus, if a ligand-induced conformational change produces a rearrangement that alters the microenvironment of an attached fluorophore, this may be reported as a fluorescence change. Detecting a conformational change with this technique is one thing: it is another matter entirely to resolve its structural basis. Indeed, the authors are not aware of any study to date that has successfully identified the structural basis of any conformational change as detected by a single fluorescent reporter.

The main advantage of VCF over conventional electrophysiology is that it offers a means of quantitating conformational changes occurring in real time at a domain of interest, distant from the pore, on the external protein surface. Specifically, VCF can provide information about (1) the temporal resolution of domain movements during gating in voltage-gated channels, (2) whether all subunits (e.g., liganded and unliganded) respond similarly during ligand-induced activation, (3) whether different ligands produce different conformational changes in a given subunit. One of the limitations of VCF is that it is difficult to unequivocally determine whether a detected conformational change is part of the gating process or whether it is detecting a peripheral event that does not contribute to the "conformational wave" that induces activation. Single channel kinetic analysis (i.e., patch clamp electrophysiology) provides a definitive means of assessing the contribution of a residue to the channel gating mechanism (Auerbach, 2010).

VCF was recently employed in our laboratory to compare glycine- and ivermectin-induced conformational changes in the al GlyR to address two questions: (1) does ivermectin elicit a global conformational change that extends into the ECD and perhaps to the glycine binding site? and (2) are these conformational changes distinct from those elicited by glycine? We investigated 12 cysteine-substituted sites throughout the $\alpha 1$ GlyR ECD that were all known to produce glycine-mediated conformational changes when labeled with rhodamine derivatives (Wang and Lynch, 2012). Most (9/12) of these labeled sites, including those located in glycine binding domain loops $\mathrm{C}$, E, and F (Figure 1C), exhibited robust fluorescence changes in response to glycine binding but no detectable fluorescence change in response 
to ivermectin binding. An example of a differential fluorescence response for the H201C mutant GlyR is shown in Figure 4A. However, at the remaining three labeled sites (N203C in binding domain loop C, Q67C in binding domain loop D, and V178C in binding domain loop F), ivermectin and glycine both elicited detectable fluorescence changes (Figure 4B). Moreover, it has previously been shown that the L233W mutation converts ivermectin into an antagonist of glycine-gated currents (Lynagh et al., 2011). As shown in Figure 4C, ivermectin-induced a fluorescence

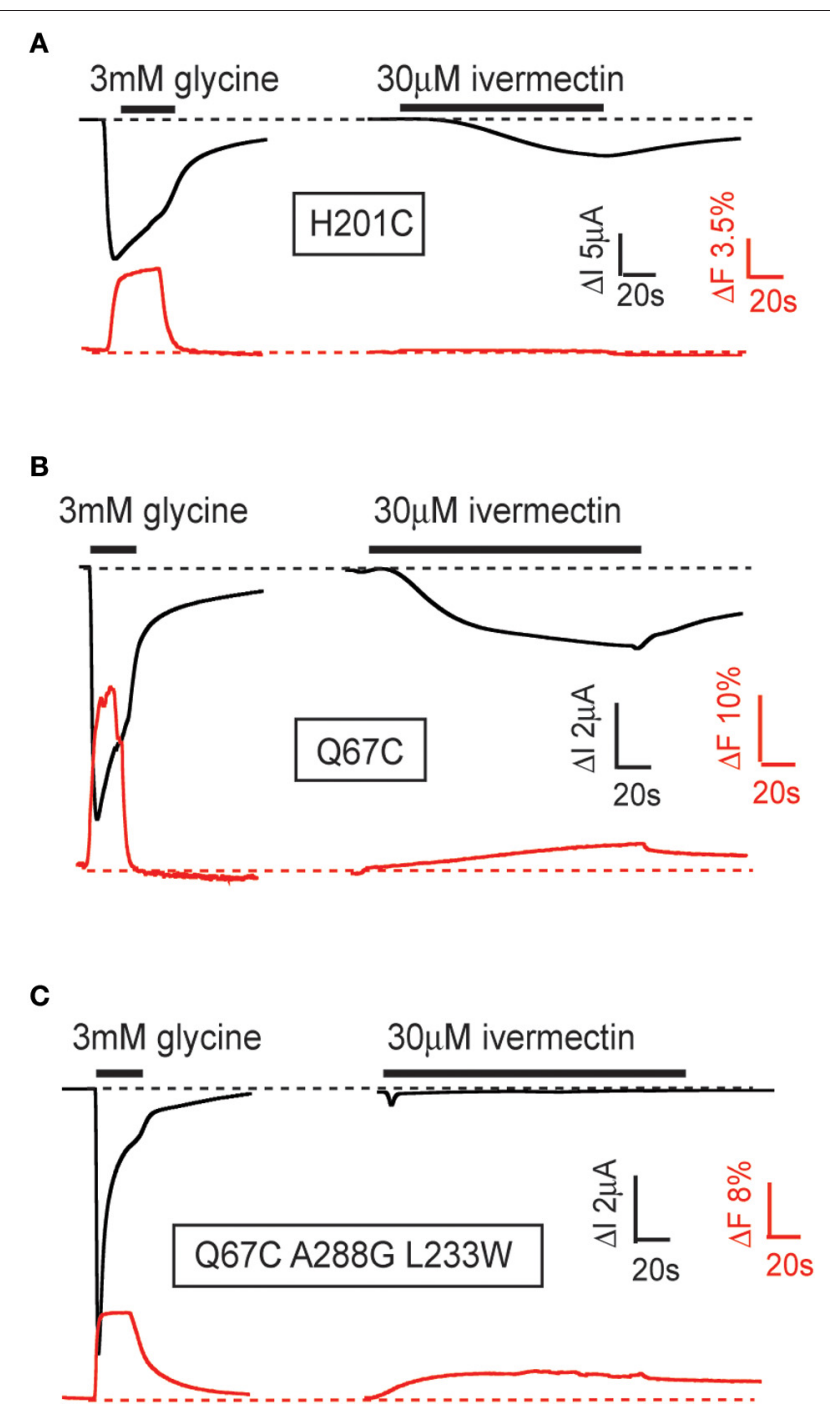

FIGURE 4 | Examples of glycine- and ivermectin-mediated fluorescence and current responses in mutant GlyRs. Current responses (black) and fluorescence responses (red) were recorded simultaneously from Xenopus oocytes expressing indicated $\alpha 1$ GlyRs. (A) Fluorescence responses induced by glycine, but not ivermectin, in fluorescently labeled H201C mutant GlyRs. (B) Fluorescence responses induced by glycine and ivermectin in fluorescently labeled Q67C mutant GlyRs. (C) Fluorescence responses induced by glycine and ivermectin in fluorescently labeled Q67C, A288G, L233W triple-mutant GlyRs. These mutations convert ivermectin into an antagonist of glycine-gated currents. The figure is modified from Wang and Lynch (2012). Please refer to that paper for full experimental details. response at the N203C-L233W double mutant GlyR indicating that ivermectin elicits a global conformational change even when it inhibits glycine currents. These results indicate that ivermectin induces conformational changes throughout the entire receptor, despite its binding site being located immediately adjacent to the M2 domain which houses the pore gate. By eliciting conformational changes in the neurotransmitter binding site, the results provide a possible explanation as to how ivermectin potentiates neurotransmitter-gated currents (Cully et al., 1994) and permits glutamate to activate the otherwise glutamate-insensitive C. elegans a GluClR (Etter et al., 1996; Hibbs and Gouaux, 2011).

\section{CONCLUSIONS}

Ivermectin binds to the TMD of Cys-loop receptors in a cleft formed at the interface between adjacent subunits. A crystal structure of ivermectin binding to the $C$. elegans $\alpha$ GluClR revealed the existence of hydrogen bonds linking ivermectin and the transmembrane residues, L218, S260, and T285. However, sitedirected mutagenesis studies and amino acid sequence analysis on other ivermectin-sensitive anionic Cys-loop receptors revealed that hydrogen bonds with residues equivalent to S260 and T285 are not required for high ivermectin sensitivity at either the $\alpha 1$ GlyR or three other high ivermectin-affinity GluClRs. As the hydrogen bond between the ivermectin C7-hydroxyl with the L218 or equivalent residues is via the protein backbone carbonyl, its existence cannot be readily tested via site-directed mutagenesis. However, this putative interaction requires confirmation given that the ivermectin C7-hydroxyl is also close enough to the carbonyl oxygen at the ivermectin $\mathrm{C} 1$ position to form an intra-molecular hydrogen bond.

The slowly reversible (or irreversible) agonist activity of ivermectin is likely to be due to it accumulating in the membrane and binding weakly (i.e., reversibly) to its site. An M3 domain glycine residue at the mouth of the ivermectin binding cleft is essential for low nanomolar ivermectin potency, and indeed a glycine at this position appears to be necessary and sufficient to confer high ivermectin potency to anionic Cys-loop receptors. Despite its functional importance, it is unlikely that bound ivermectin has a significant energetic interaction with this residue when bound to its site. It is more likely that side chains larger than glycine form a physical barrier that inhibits ivermectin from efficiently accessing its site in the cleft. Finally, ivermectin binding opens the channel pore via a structural change that is different to that induced by the endogenous neurotransmitter agonist. However, ivermectin does simultaneously induce a global conformational change that propagates to the neurotransmitter binding site, thus suggesting one mechanism by which ivermectin may potentiate neurotransmitter-gated currents. Together, this information provides important insights into the mechanisms of action of this gold standard anthelmintic drug.

\section{ACKNOWLEDGMENTS}

Research in the authors' laboratory is supported by the Australian Research Council and the National Health and Medical Research Council of Australia (NHMRC). Joseph W. Lynch is supported by an NHMRC Senior Research Fellowship. 


\section{REFERENCES}

Adelsberger, H., Lepier, A., and Dudel, J. (2000). Activation of rat recombinant alpha(1)beta(2)gamma(2S) GABA(A) receptor by the insecticide ivermectin. Eur. J. Pharmacol. 394, 163-170.

Akabas, M. H., Kaufmann, C., Archdeacon, P., and Karlin, A. (1994). Identification of acetylcholine receptor channel-lining residues in the entire $\mathrm{M} 2$ segment of the alpha subunit. Neuron 13, 919-927.

Amin, J., and Weiss, D. S. (1993). GABAA receptor needs two homologous domains of the beta-subunit for activation by GABA but not by pentobarbital. Nature 366, 565-569.

Auerbach, A. (2010). The gating isomerization of neuromuscular acetylcholine receptors. J. Physiol. 588, 573-586

Beg, A. A., and Jorgensen, E. M. (2003). EXP-1 is an excitatory GABA-gated cation channel. Nat. Neurosci. 6, 1145-1152.

Bloom, R. A., and Matheson, J. C. 3rd. (1993). Environmental assessment of avermectins by the US Food and Drug Administration. Vet. Parasitol. 48, 281-294

Bocquet, N., Nury, H., Baaden, M., Le Poupon, C., Changeux, J. P., Delarue, M., and Corringer, P. J. (2009). X-ray structure of a pentameric ligand-gated ion channel in an apparently open conformation. Nature 457, 111-114.

Brejc, K., van Dijk, W. J., Klaassen, R. V., Schuurmans, M., van Der Oost, I., Smit, A. B., and Sixma, T. K. (2001). Crystal structure of an AChbinding protein reveals the ligandbinding domain of nicotinic receptors. Nature 411, 269-276.

Brown, D. D., Siddiqui, S. Z., Kaji, M. D., and Forrester, S. G. (2012). Pharmacological characterization of the Haemonchus contortus GABA-gated chloride channel, Hco-UNC-49: modulation by macrocyclic lactone anthelmintics and a receptor for piperazine. Vet. Parasitol. 185, 201-209.

Changeux, J. P. (2010). Allosteric receptors: from electric organ to cognition. Annu. Rev. Pharmacol. Toxicol. 50, 1-38.

Chiara, D. C., Dostalova, Z., Jayakar, S. S., Zhou, X., Miller, K. W., and Cohen, J. B. (2012). Mapping general anesthetic binding site(s) in human alphalbeta3 gammaaminobutyric acid type $\mathrm{A}$ (GABAA) receptors with $\left[{ }^{3} \mathrm{H}\right]$ TDBzletomidate, a photoreactive etomidate analog. Biochemistry $51,836-847$.
Collingridge, G. L., Olsen, R. W., Peters, J., and Spedding, M. (2009). A nomenclature for ligand-gated ion channels. Neuropharmacology 56, 2-5.

Collins, T., and Millar, N. S. (2010) Nicotinic acetylcholine receptor transmembrane mutations convert ivermectin from a positive to a negative allosteric modulator. $\mathrm{Mol}$. Pharmacol. 78, 198-204.

Costa, J. L., and Diazgranados, J. A. (1994). Ivermectin for spasticity in spinal-cord injury. Lancet 343, 739.

Crump, A., and Omura, S. (2011). Ivermectin, 'wonder drug' from Japan: the human use perspective. Proc. Jpn. Acad. Ser. B Phys. Biol. Sci. $87,13-28$.

Cully, D. F., Paress, P. S., Liu, K K., Schaeffer, J. M., and Arena, J. P. (1996). Identification of a Drosophila melanogaster glutamate-gated chloride channel sensitive to the antiparasitic agent avermectin. J. Biol. Chem. 271, 20187-20191.

Cully, D. F., Vassilatis, D. K., Liu, K. K., Paress, P. S., van der Ploeg, L. H., Schaeffer, J. M., and Arena, J. P. (1994). Cloning of an avermectinsensitive glutamate-gated chloride channel from Caenorhabditis elegans. Nature 371, 707-711.

Cymes, G. D., and Grosman, C. (2011). Tunable pKa values and the basis of opposite charge selectivities in nicotinic-type receptors. Nature $474,526-530$

Cymes, G. D., Ni, Y., and Grosman, C. (2005). Probing ion-channel pores one proton at a time. Nature 438 , 975-980.

Eichler, S. A., Forstera, B., Smolinsky, B., Juttner, R., Lehmann, T. N. Fahling, M., Schwarz, G., Legendre, P., and Meier, J. C. (2009). Splicespecific roles of glycine receptor alpha3 in the hippocampus. Eur. J. Neurosci. 30, 1077-1091.

Etter, A., Cully, D. F., Schaeffer, J. M., Liu, K. K., and Arena, J. P. (1996). An amino acid substitution in the pore region of a glutamate-gated chloride channel enables the coupling of ligand binding to channel gating. J. Biol. Chem. 271, 16035-16039.

Forrester, S. G., Prichard, R. K., Dent, J. A., and Beech, R. N. (2003). Haemonchus contortus: HcGluCla expressed in Xenopus oocytes forms a glutamate-gated ion channel that is activated by ibotenate and the antiparasitic drug ivermectin. Mol. Biochem. Parasitol. 129, 115-121.

Fritz, L. C., Wang, C. C., and Gorio, A. (1979). Avermectin B1a irreversibly blocks postsynaptic potentials at the lobster neuromuscular junction by reducing muscle membrane resistance. Proc. Natl. Acad. Sci. U.S.A 76, 2062-2066.

Galzi, J. L., Bertrand, D., DevillersThiery, A., Revah, F., Bertrand, S., and Changeux, J. P. (1991). Functional significance of aromatic amino acids from three peptide loops of the alpha 7 neuronal nicotinic receptor site investigated by site-directed mutagenesis. FEBS Lett. 294, 198-202.

Galzi, J. L., Devillers-Thiery, A., Hussy, N., Bertrand, S., Changeux, J. P. and Bertrand, D. (1992). Mutations in the channel domain of a neuronal nicotinic receptor convert ion selectivity from cationic to anionic. Nature 359, 500-505.

Gandhi, C. S., and Isacoff, E. Y. (2005) Shedding light on membrane proteins. Trends Neurosci. 28, 472-479.

Ghosh, R., Andersen, E. C., Shapiro, J. A., Gerke, J. P., and Kruglyak, L. (2012). Natural variation in a chloride channel subunit confers avermectin resistance in $C$. elegans. Science 335, 574-578.

Grudzinska, J., Schemm, R., Haeger, S. Nicke, A., Schmalzing, G., Betz, H., and Laube, B. (2005). The $\beta$ subunit determines the ligand binding properties of synaptic glycine receptors. Neuron 45, 727-739.

Grutter, T., de Carvalho, L. P., Dufresne, V., Taly, A., Edelstein, S. J., and Changeux, J. P. (2005). Molecular tuning of fast gating in pentameric ligand-gated ion channels. Proc. Natl. Acad. Sci. U.S.A. 102, 18207-18212.

Hansen, S. B., Sulzenbacher, G., Huxford, T., Marchot, P., Taylor, P., and Bourne, Y. (2005). Structures of Aplysia AChBP complexes with nicotinic agonists and antagonists reveal distinctive binding interfaces and conformations. EMBO J. 24, 3635-3646

Hazai, E., Joshi, P., Skoviak, E. C., Suryanarayanan, A., Schulte, M. K., and Bikadi, Z. (2009). A comprehensive study on the 5-hydroxytryptamine(3A) receptor binding of agonists serotonin and $\mathrm{m}$-chlorophenylbiguanidine. Bioorg. Med. Chem. 17, 5796-5805.

Hibbs, R. E., and Gouaux, E. (2011) Principles of activation and permeation in an anion-selective Cys-loop receptor. Nature 474, 54-60.

Hilf, R. J., and Dutzler, R. (2008). $\mathrm{X}$-ray structure of a prokaryotic pentameric ligand-gated ion channel. Nature 452, 375-379.

Hilf, R. J., and Dutzler, R. (2009) Structure of a potentially open state of a proton-activated pentameric ligand-gated ion channel. Nature $457,115-118$.

Horenstein, J., Wagner, D. A., Czajkowski, C., and Akabas, M. H. (2001). Protein mobility and GABA-induced conformational changes in $\mathrm{GABA}(\mathrm{A})$ receptor porelining M2 segment. Nat. Neurosci. 4, $477-485$.

Huang, J., and Casida, J. E. (1997) Avermectin Bla binds to high- and low-affinity sites with dual effects on the gamma-aminobutyric acidgated chloride channel of cultured cerebellar granule neurons. J. Pharmacol. Exp. Ther. 281, 261-266.

Imoto, K., Busch, C., Sakmann, B., Mishina, M., Konno, T., Nakai, J., Bujo, H., Mori, Y., Fukuda, K., and Numa, S. (1988). Rings of negatively charged amino acids determine the acetylcholine receptor channel conductance. Nature 335, 645-648.

Jones, A. K., Bera, A. N., Lees, K. and Sattelle, D. B. (2010). The cysloop ligand-gated ion channel gene superfamily of the parasitoid wasp, Nasonia vitripennis. Heredity 104, 247-259.

Jones, A. K., and Sattelle, D. B. (2008). The cys-loop ligand-gated ion channel gene superfamily of the nematode, Caenorhabditis elegans. Invert. Neurosci. 8, 41-47.

Kane, N. S., Hirschberg, B., Qian, S., Hunt, D., Thomas, B., Brochu, R., Ludmerer, S. W., Zheng, Y., Smith, M., Arena, I. P., Cohen, C. J., Schmatz, D., Warmke, J., and Cully, D. F. (2000). Drug-resistant Drosophila indicate glutamategated chloride channels are targets for the antiparasitics nodulisporic acid and ivermectin. Proc. Natl. Acad. Sci. U.S.A. 97, 13949-13954.

Kash, T. L., Jenkins, A., Kelley, J. C., Trudell, J. R., and Harrison, N. L. (2003). Coupling of agonist binding to channel gating in the $\operatorname{GABA}(\mathrm{A})$ receptor. Nature 421, 272-275.

Kass, I. S., Wang, C. C., Walrond, J. P., and Stretton, A. O. (1980) Avermectin Bla, a paralyzing anthelmintic that affects interneurons and inhibitory motoneurons in Ascaris. Proc. Natl. Acad. Sci. U.S.A. 77, 6211-6215.

Kawate, T., Michel, J. C., Birdsong, W. T., and Gouaux, E. (2009). Crystal structure of the ATP-gated P2X(4) ion channel in the closed state. Nature 460, 592-598.

Kehoe, J., Buldakova, S., Acher, F. Dent, J., Bregestovski, P., and Bradley, J. (2009). Aplysia cys-loop glutamate-gated chloride channels reveal convergent evolution of ligand specificity. J. Mol. Evol. 69, 125-141. 
Keramidas, A., Moorhouse, A. J., French, C. R., Schofield, P. R., and Barry, P. H. (2000). M2 pore mutations convert the glycine receptor channel from being anionto cation-selective. Biophys. J. 79, 247-259.

Keramidas, A., Moorhouse, A. J., Pierce, K. D., Schofield, P. R., and Barry, P. H. (2002). Cation-selective mutations in the M2 domain of the inhibitory glycine receptor channel reveal determinants of ion-charge selectivity. J. Gen. Physiol. 119, 393-410.

Keramidas, A., Moorhouse, A. J., Schofield, P. R., and Barry, P. H. (2004). Ligand-gated ion channels: mechanisms underlying ion selectivity. Prog. Biophys. Mol. Biol. 86, 161-204.

Khakh, B. S., Proctor, W. R., Dunwiddie, T. V., Labarca, C., and Lester, H. A. (1999). Allosteric control of gating and kinetics at $\mathrm{P} 2 \mathrm{X}(4)$ receptor channels. J. Neurosci. 19, 7289-7299.

Krause, R. M., Buisson, B., Bertrand, S., Corringer, P. J., Galzi, J. L., Changeux, J. P., and Bertrand, D. (1998). Ivermectin: a positive allosteric effector of the alpha7 neuronal nicotinic acetylcholine receptor. Mol. Pharmacol. 53, 283-294.

Kwon, D. H., Yoon, K. S., Clark, J. M., and Lee, S. H. (2010). A point mutation in a glutamate-gated chloride channel confers abamectin resistance in the two-spotted spider mite, Tetranychus urticae Koch. Insect. Mol. Biol. 19, 583-591.

Lee, S. Y., and MacKinnon, R. (2004). A membrane-access mechanism of ion channel inhibition by voltage sensor toxins from spider venom. Nature 430, 232-235.

Lee, W. Y., and Sine, S. M. (2005). Principal pathway coupling agonist binding to channel gating in nicotinic receptors. Nature 438, 243-247.

Lerchner, W., Xiao, C., Nashmi, R., Slimko, E. M., van Trigt, L., Lester, H. A., and Anderson, D. J. (2007). Reversible silencing of neuronal excitability in behaving mice by a genetically targeted, ivermectingated Cl- channel. Neuron 54, 35-49.

Li, G. D., Chiara, D. C., Sawyer, G. W., Husain, S. S., Olsen, R. W., and Cohen, J. B. (2006). Identification of a GABAA receptor anesthetic binding site at subunit interfaces by photolabeling with an etomidate analog. J. Neurosci. 26, 11599-11605.

Li, P., Slimko, E. M., and Lester, H. A. (2002). Selective elimination of glutamate activation and introduction of fluorescent proteins into a Caenorhabditis elegans chloride channel. FEBS Lett. 528, 77-82.

Lynagh, T., and Lynch, J. W. (2010a) A glycine residue essential for high ivermectin sensitivity in Cysloop ion channel receptors. Int. J. Parasitol. 40, 1477-1481.

Lynagh, T., and Lynch, J. W. (2010b). An improved ivermectin-activated chloride channel receptor for inhibiting electrical activity in defined neuronal populations. J. Biol. Chem. 285, 14890-14897.

Lynagh, T., Webb, T. I., Dixon, C. L. Cromer, B. A., and Lynch, J. W. (2011). Molecular determinants of ivermectin sensitivity at the glycine receptor chloride channel. J. Biol. Chem. 286, 43913-43924.

Martin, R. J., and Kusel, J. R. (1992). On the distribution of a fluorescent ivermectin probe (4" 5,7 dimethylbodipy proprionylivermectin) in Ascaris membranes. Parasitology 104(Pt 3), 549-555.

Martin, R. J., and Pennington, A. J. (1989). A patch-clamp study of effects of dihydroavermectin on Ascaris muscle. Br. J. Pharmacol. 98, 747-756.

McCavera, S., Rogers, A. T., Yates, D. M., Woods, D. J., and Wolstenholme, A. J. (2009). An ivermectin-sensitive glutamategated chloride channel from the parasitic nematode Haemonchus contortus. Mol. Pharmacol. 75, 1347-1355.

Michael, B., Meinke, P. T., and Shoop, W. (2001). Comparison of ivermectin, doramectin, selamectin, and eleven intermediates in a nematode larval development assay. $J$. Parasitol. 87, 692-696.

Miller, P. S., and Smart, T. G. (2010) Binding, activation and modulation of Cys-loop receptors. Trends Pharmacol. Sci. 31, 161-174.

Miyazawa, A., Fujiyoshi, Y., and Unwin, N. (2003). Structure and gating mechanism of the acetylcholine receptor pore. Nature 423, 949-955.

Mortensen, M., and Smart, T. G. (2007). Single-channel recording of ligand-gated ion channels. Nat Protoc. 2, 2826-2841.

Njue, A. I., Hayashi, J., Kinne, L., Feng, X. P., and Prichard, R. K. (2004). Mutations in the extracellular domains of glutamate-gated chloride channel alpha3 and beta subunits from ivermectin-resistant Cooperia oncophora affect agonist sensitivity. J. Neurochem. 89, 1137-1147.

Omura, S. (2008). Ivermectin: 25 years and still going strong. Int. J. Antimicrob. Agents 31, 91-98.
Paas, Y., Gibor, G., Grailhe, R., SavatierDuclert, N., Dufresne, V., Sunesen, M., de Carvalho, L. P., Changeux J. P., and Attali, B. (2005). Pore conformations and gating mechanism of a Cys-loop receptor. Proc. Natl. Acad. Sci. U.S.A. 102, 15877-15882.

Pistis, M., Belelli, D., Peters, J. A., and Lambert, J. J. (1997). The interaction of general anaesthetics with recombinant GABAA and glycine receptors expressed in Xenopus laevis oocytes: a comparative study. Br. J. Pharmacol. 122, 1707-1719.

Pless, S. A., Dibas, M. I., Lester, H. A., and Lynch, J. W. (2007) Conformational variability of the glycine receptor M2 domain in response to activation by different agonists. J. Biol. Chem. 282, 36057-36067.

Pless, S. A., Leung, A. W., Galpin, J. D., and Ahern, C. A. (2011) Contributions of conserved residues at the gating interface of glycine receptors. J. Biol. Chem. 286, 35129-35136.

Pless, S. A., and Lynch, J. W. (2008). Illuminating the structure and function of Cys-loop receptors. Clin. Exp. Pharmacol. Physiol. 35 1137-1142.

Pless, S. A., and Lynch, J. W. (2009) Ligand-specific conformational changes in the alphal glycine receptor ligand-binding domain. J. Biol. Chem. 284, 15847-15856.

Pong, S. S., and Wang, C. C. (1982) Avermectin B1a modulation of gamma-aminobutyric acid receptors in rat brain membranes. $J$ Neurochem. 38, 375-379.

Purohit, P., Mitra, A., and Auerbach, A. (2007). A stepwise mechanism for acetylcholine receptor channel gating. Nature 446, 930-933.

Rajendra, S., Vandenberg, R. J., Pierce, K. D., Cunningham, A. M., French P. W., Barry, P. H., and Schofield, P. R. (1995). The unique extracellular disulfide loop of the glycine receptor is a principal ligand binding element. EMBO J. 14 , 2987-2998.

Ranganathan, R., Cannon, S. C., and Horvitz, H. R. (2000). MOD-1 is a serotonin-gated chloride channel that modulates locomotory behaviour in C. elegans. Nature 408 470-475.

Raymond, V., Mongan, N. P., and Sattelle, D. B. (2000). Anthelmintic actions on homomer-forming nicotinic acetylcholine receptor subunits: chicken alpha7 and ACR-16 from the nematode Caenorhabditis elegans. Neuroscience 101, 785-791.
Rudolph, U., and Antkowiak, B. (2004). Molecular and neuronal substrates for general anaesthetics. Nat. Rev. Neurosci. 5, 709-720.

Schnizler, K., Saeger, B., Pfeffer, C., Gerbaulet, A., EbbinghausKintscher, U., Methfessel, C., Franken, E. M., Raming, K. Wetzel, C. H., Saras, A., Pusch, H., Hatt, H., and Gisselmann, G. (2005). A novel chloride channel in Drosophila melanogaster is inhibited by protons. J. Biol. Chem. 280, 16254-16262.

Shan, Q., Haddrill, J. L., and Lynch, J. W. (2001). Ivermectin, an unconventional agonist of the glycine receptor chloride channel. J. Biol. Chem. 276, 12556-12564.

Silberberg, S. D., Li, M., and Swartz, K. J. (2007). Ivermectin Interaction with transmembrane helices reveals widespread rearrangements during opening of P2X receptor channels. Neuron 54, 263-274.

Taly, A., Delarue, M., Grutter, T., Nilges, M., Le Novere, N., Corringer, P. J., and Changeux, J. P. (2005). Normal mode analysis suggests a quaternary twist model for the nicotinic receptor gating mechanism. Biophys. J. 88, 3954-3965.

Thompson, A. J., Lester, H. A., and Lummis, S. C. (2010). The structural basis of function in Cysloop receptors. Q. Rev. Biophys. 43, 449-499.

Tsukamoto, Y., Sato, K., Mio, S., Sugai, S., Yanai, T., Kitano, N., Muramatsu, S., Nakada, Y., and Ide, J. (1991). Synthesis of 5keto-5-oxime derivatives of milbemycins and their activities against microfilariae. Agric. Biol. Chem. 55, 2615-2621.

Unwin, N. (1995). Acetylcholine receptor channel imaged in the open state. Nature 373, 37-43.

Unwin, N. (2005). Refined structure of the nicotinic acetylcholine receptor at $4 \mathrm{~A}$ resolution. J. Mol. Biol. 346, 967-989.

Wagner, D. A., and Czajkowski, C. (2001). Structure and dynamics of the GABA binding pocket: a narrowing cleft that constricts during activation. J. Neurosci. 21, $67-74$.

Wang, H. L., Cheng, X., and Sine, S. M. (2012). Intra-membrane proton binding site linked to activation of a bacterial pentameric ion channel. J. Biol. Chem. 287, 6482-6489.

Wang, Q., and Lynch, J. W. (2012). A comparison of glycine- and ivermectin-mediated conformational changes in the glycine 
receptor ligand-binding domain. Int. J. Biochem. Cell Biol. 44, 335-340.

Webb, T. I., and Lynch, J. W. (2007). Molecular pharmacology of the glycine receptor chloride channel. Curr. Pharm. Des. 13, 2350-2367.

Wolstenholme, A. J. (2010). Recent progress in understanding the interaction between avermectins and ligand-gated ion channels: putting the pests to sleep. Invert. Neurosci. 10, 5-10.

Wolstenholme, A. J., Fairweather, I., Prichard, R., von SamsonHimmelstjerna, G., and Sangster, N. C. (2004). Drug resistance in veterinary helminths. Trends Parasitol. 20, 469-476.
Wolstenholme, A. J., and Rogers, A. T. (2005). Glutamate-gated chloride channels and the mode of action of the avermectin/milbemycin anthelmintics. Parasitology 131 (Suppl), S85-S95.

Xiu, X., Hanek, A. P., Wang, J., Lester, H. A., and Dougherty, D. A. (2005). A unified view of the role of electrostatic interactions in modulating the gating of Cys loop receptors. J. Biol. Chem. 280, 41655-41666.

Yamaguchi, M., Sawa, Y., Matsuda, K., Ozoe, F., and Ozoe, Y. (2012) Amino acid residues of both the extracellular and transmembrane domains influence binding of the antiparasitic agent milbemycin to Haemonchus contortus AVR-14B glutamate-gated chloride channels. Biochem. Biophys. Res. Commun. 419, 562-566.

Yevenes, G. E., and Zeilhofer, H. U. (2011). Allosteric modulation of glycine receptors. Br. J. Pharmacol. 164, 224-236.

Zheng, Y., Hirschberg, B., Yuan, J. Wang, A. P., Hunt, D. C., Ludmerer S. W., Schmatz, D. M., and Cully, D. F. (2002). Identification of two novel Drosophila melanogaster histamine-gated chloride channe subunits expressed in the eye. J. Biol. Chem. 277, 2000-2005.

Conflict of Interest Statement: The authors declare that the research was conducted in the absence of any commercial or financial relationships that could be construed as a potentia conflict of interest.

Received: 06 March 2012; accepted: 17 April 2012; published online: 07 May 2012.

Citation: Lynagh T and Lynch JW (2012) Molecular mechanisms of Cys-loop ion channel receptor modulation by ivermectin. Front. Mol. Neurosci. 5:60. doi: 10.3389/fnmol.2012.00060

Copyright (c) 2012 Lynagh and Lynch. This is an open-access article distributed under the terms of the Creative Commons Attribution Non Commercial License, which permits non-commercial use, distribution, and reproduction in other forums, provided the original authors and source are credited. 